\title{
Etude du transfert du radon 222 à l'intérieur des habitations ${ }^{\star}$
}

\author{
R. MAXIMILIEN', M.C. ROBE 2 , M. ARCHIMBAUD ${ }^{3}$ \\ (Manuscrit reçu le 3 décembre 1984)
}

\begin{abstract}
RÉSUME
L'exposition domestique est susceptible de varier considerablement suivant l'environnement naturel (géologie, climat), les aménagements humains (matériaux de construction, systèmes d'isolation et de ventilation,...) ou encore les habitudes de vie. Dans une habitation donnée, la mise en évidence des sources et l'évaluation de leur contribution respective exige une connaissance précise des processus de transfert et de dispersion du radon et nécessite, par voie de conséquence, la mise en jeu d'un dispositif expérimental lourd (mesure continue du radon, de la ventilation,...). Elle doit être réservée à l'étude de cas sélectionnés par un programme de mesure systématique, soit pour leur représentativité de l'habitat, soit de préférence pour leur forte teneur en radon dont elle cherchera alors à expliquer l'origine. Une retombée éventuelle de ce travail est d'aider à la mise au point de contremesures.
\end{abstract}

Cet exposé a pour objet de présenter une étude pilote du transfert de radon sur deux maisons individuelles de la vallée du Rhône. Bien que les deux habitations sélectionnées parmi 131 pour leur teneur élevée en radon soient construites sur un même mode architectural et situées à proximité immédiate l'une de l'autre, les facteurs explicatifs de l'exposition domestique y diffèrent sensiblement. Les paramètres intérieurs à l'habitation induisent des variations de concentration de radon dans le cas d'une faible ventilation naturelle. Au contraire, seuls les paramètres extérieurs semblent exercer une influence en cas de ventilation importante. Cependant, le radon semble provenir pour une grande part du sol dans ces deux habitations.

\section{ABSTRACT}

Indoor exposure can vary considerably depending upon the natural environment (geology, climate), man-made arrangements (building

* Communication présentée lors du séminaire organisé par la Société française de radioprotection sur "les données actuelles sur la radioactivité naturelle", Monte-Carlo, 5-7 novembre 1984.

1. Commissariat à l'énergie atomique, Institut de protection et de sûreté nucléaire, DPS/GMR CE, BP 6, 92260 Fontenay-aux-Roses, France.

2. Commissariat à l'énergie atomique, Institut de protection et de sûreté nucléaire, DPT/SPIN, 91191 Gif-sur-Yvette Cedex, France.

3. Commissariat à l'énergie atomique, Institut de protection et de sûreté nucléaire, DPS/SHI, BP 16, 26701 Pierrelatte Cedex, France. 
materials, insulation and ventilation systems,...) or way of living. In order to specify the sources and assess their respective contribution in a given dwelling, a good knowledge of radon transfer and dispersion processes is required as well as a heavy experimental device (continuous radon and ventilation monitoring,...). The study must be limited to some cases selected by a systematic measurement program either because they are representative of dwelling conditions, or preferably on account of their high radon level, the origin of which will be investigated. As a consequence, countermeasures can be developed.

A pilot study has been carried out on radon transport in two houses of the Rhone river valley. The two houses - selected among 131 other ones for their high radon levels - are built with the same architectural approach and located very close to each other, yet the factors accounting for domestic exposure are quite different. Indoor parameters are at the origin of various radon concentrations in the case of low natural ventilation ; conversely, outdoor parameters only seem to act in the case of high ventilation. For a larger part, however, radon seems to emanate from under the foundations of both houses.

\section{INTRODUCTION}

La concentration de radon 222 et de ses descendants à courte période dans l'air des habitations dépend non seulement de l'émanation de radon 222 à partir de sources (matériaux de construction, sol sous-jacent, consommation d'eau et de gaz naturel) et de la concentration en radon 222 de l'air extérieur, mais aussi des conditions météorologiques, de la ventilation des locaux et des habitudes de vie des occupants. Cet ensemble complexe de paramètres explique l'importante variabilité des teneurs en radon 222 observées d'un bâtiment à l'autre, et, pour un même bâtiment, d'un moment à l'autre. En dépit de la multiplication récente des investigations sur ce problème, on ne dispose pas actuellement de modèles satisfaisants pour rendre compte de toutes les fluctuations possibles de l'exposition domestique tant en fonction des localisations géographiques que des caractéristiques architecturales de l'habitat ou encore des facteurs climatiques.

Les campagnes de mesure systématique du radon 222 ou de ses descendants ont pour objet essentiel de déterminer la distribution des niveaux moyens d'activité de ces nucléides dans le milieu domestique. Ayant vocation d'enquêtes à grande échelle, elles mettent en œuvre des moyens de mesure réduits et autonomes consistant habituellement en des détecteurs passifs de traces $[2,9,14,16]$ et, plus rarement, en des dosimètres "actifs" qui assurent une mesure de l'énergie alpha potentielle des descendants à vie courte du radon [3]. Leurs résultats, qui expriment l'activité moyenne (ou l'énergie) observée au cours d'un intervalle de temps donné, généralement un mois, permettent d'analyser l'influence de variables grossières (géologie, structure de l'habitat, saisons,...).

Dans une habitation donnée, la mise en évidence des sources et l'évaluation de leurs contributions respectives exigent une connaissance précise des processus de transfert et de dispersion du radon 222 . L'établissement de rapports entre les sources et la concentration en radon 222 
de l'air ambiant repose donc sur le contrôle simultané de leurs variables et nécessite, par voie de conséquence, la mise en jeu d'un dispositif expérimental important. De telles études ne peuvent porter que sur un échantillon limité, soit représentatif de l'habitat local, soit sélectionné pour sa forte teneur en radon 222 ; dans cette dernière éventualité, l'étude pourra servir à mettre au point des moyens de limitation et à vérifier leur efficacité.

\section{CONSIDERATIONS GENERALES SUR LA VARIABILITE DES NIVEAUX DE RADON 222 DANS LES HABITATIONS : L'IMPORTANCE DES MECANISMES DE TRANSFERT}

L'analyse de la variabilité des niveaux d'exposition domestique repose sur l'établissement d'un bilan des entrées et des sorties de radon des habitations en cherchant à en caractériser les variables. De ce point de vue, deux paramètres conditionnent au premier chef la concentration en radon 222 du milieu intérieur : l'émanation de radon 222 à partir des sources et le taux de renouvellement de l'air des locaux.

\subsection{L'influence de la ventilation}

On peut considérer sans trop grand risque d'erreur que la ventilation constitue le seul mécanisme de sortie du radon 222 des habitations. L'établissement d'une relation entre la teneur en radon de l'air et la ventilation ne pose pas de difficulté particulière lorsqu'on mesure ces deux paramètres de façon continue. En règle générale, la concentration de radon 222 varie de façon inversement proportionnelle au taux de renouvellement de l'air, à condition de supposer une distribution immédiate et homogène du radon 222 dans l'espace étudié. On dispose de nombreuses données expérimentales à l'appui de cette notion [17, 18].

Lorsqu'on déborde de ce cadre expérimental, l'évaluation de la ventilation moyenne et de son influence sur le niveau moyen de radon 222 (mesures systématiques) devient beaucoup plus délicate. Toute la difficulté réside dans le choix d'une valeur numérique représentative du renouvellement moyen de l'air de l'habitation puisque certaines variables, comme les habitudes de vie des occupants, ne peuvent être contrôlées. Ce problème revêt une importance d'autant plus grande que la ventilation constitue l'un des facteurs déterminants de l'état d'équilibre entre le radon 222 et ses descendants à vie courte qui sont les réels responsables des effets radiologiques.

\subsection{L'influence des sources}

La variabilité du niveau de radon 222 dans les habitations tient pour une grande part à la diversité des sources en présence.

On peut distinguer des sources intérieures, constituées par les matériaux de construction et le sol sous-jacent au bâtiment et des sources extérieures liées à la consommation d'eau ou de gaz riches en radon 222 ou encore à l'entrée d'air extérieur chargé en radon 222. 


\subsubsection{Les sources intérieures}

D'une façon générale, les sols et les matériaux de construction sont susceptibles d'émettre d'autant plus de radon 222 que leur teneur en radium 226 est plus forte.

Certains matériaux de construction d'origine naturelle présentent des activités volumiques de radium 226 relativement élevées : les granites, les tufs utilisés en Italie pour le gros œuvre et le revêtement des murs, et surtout les schistes alunifères employés dans la fabrication du béton aéré en Suède jusqu'en 1975. La plupart des exemples de composants riches en radium 226 proviennent de l'utilisation de plus en plus fréquente dans l'industrie du bâtiment de résidus industriels : le phosphogypse, les scories de hauts-fourneaux, les boues rouges utilisées en Allemagne pour fabriquer des briques... [13].

En ce qui concerne les sols, on estime la plage des activités massiques des radionucléides de la chaîne de l'uranium 238 à 10-50 Bq. kg-1 [20] ; des valeurs significativement plus élevées peuvent être relevées dans des régions uranifères. Cependant, seule une fraction des atomes de radon 222 formés dans le matériau sont susceptibles de quitter le réseau cristallin pour pénétrer avant décroissance radioactive, dans l'espace des pores (ou des fissures).

On définit le taux d'émanation comme le rapport du nombre d'atomes de radon 222 qui arrivent dans l'espace des pores au nombre de ceux produits dans la structure [22]. On comprend donc la difficulté d'utiliser la seule teneur en radium 226 pour caractériser la capacité à émettre du radon 222. Le taux d'émanation peut être déterminé expérimentalement par la production effective de radon 222 par des fragments de matériau suffisamment petits pour que les phénomènes de transfert n'affectent pas la mesure.

Le tableau I met en évidence la disparité de l'émanation selon les matériaux et les échantillons d'un même matériau [12]. De même, les sols présentent des taux d'émanation variables selon leur origine, leur humidité.

TABLEAU ।

Débit d'activité massique du radon 222 - Exemples

\begin{tabular}{|c|c|c|c|}
\hline Pays & Matériaux & $\begin{array}{l}\text { Radium } 226 \\
\left(\mathrm{~Bq}^{\left.-\mathrm{kg}^{-1}\right)}\right.\end{array}$ & $\begin{array}{l}\text { Débit d'activité massique } \\
\text { du radon } 222 \\
\left(10^{-5} \mathrm{~Bq} \cdot \mathrm{kg}^{-1} \cdot \mathrm{s}^{-1}\right)\end{array}$ \\
\hline URSS & $\begin{array}{l}\text { Béton lourd } \\
\text { Béton léger } \\
\text { Briques rouges }\end{array}$ & $\begin{array}{r}66 \\
141 \\
50\end{array}$ & $\begin{array}{l}0,32 \\
0,41 \\
0,16\end{array}$ \\
\hline Hongrie & $\begin{array}{l}\text { Béton } \\
\text { Briques rouges }\end{array}$ & $\begin{array}{l}13 \\
55\end{array}$ & $\begin{array}{l}0,78 \\
0,39\end{array}$ \\
\hline USA & $\begin{array}{l}\text { Béton } \\
\text { Béton + cendres } \\
\text { volantes } \\
\text { Briques rouges } \\
\text { Gypse }\end{array}$ & $\begin{array}{r}9-32 \\
19 \\
45 \\
12\end{array}$ & $\begin{array}{c}0,43 \cdot 1,26 \\
1,04 \\
0,10 \\
0,63\end{array}$ \\
\hline
\end{tabular}




\subsubsection{Les sources extérieures}

L'eau peut, dans certaines circonstances, constituer une source non négligeable de radon 222. En règle générale, les eaux de surface contiennent des concentrations relativement faibles de radon 222 (quelques $\mathrm{kBq} \cdot \mathrm{m}^{-3}$ ) comparativement aux eaux de puits, surtout si ceux-ci ont été forés en zone granitique (plusieurs centaines, voire milliers de $\mathrm{kBq} \cdot \mathrm{m}^{-3}$ ). Compte tenu de la consommation journalière moyenne d'eau $\left(0,2\right.$ à $0,4 \mathrm{~m}^{3}$ par tête), seules des concentrations de radon 222 atteignant ou dépassant $10 \mathrm{kBq} \cdot \mathrm{m}^{-3}$ sont susceptibles de contribuer significativement à l'exposition domestique [20].

On dispose de peu de données sur l'apport domestique de radon 222 lié à la consommation de gaz naturel dont les teneurs en radon s'échelonnent de quelques Bq. $\mathrm{m}^{-3}$ à $50 \mathrm{kBq} \cdot \mathrm{m}^{-3}$.

\subsection{L'importance des phénomènes de transfert}

Comprendre la présence de radon 222 dans les habitations suppose non seulement de comprendre le processus de sortie du radon du réseau cristallin, mais aussi les phénomènes de transfert de l'espace des pores vers l'extérieur du matériau "interface source-atmosphère" ainsi que les voies de passage allant des sources vers l'intérieur de l'habitation " interfacesource-milieu intérieur".

\subsubsection{L'interface source-atmosphère}

Après libération dans l'espace des pores du sol ou des matériaux de construction, les atomes de radon 222 gagnent l'atmosphère par diffusion simple, voire sous l'influence de phénomènes de convection. Le flux d'émanation de radon 222 à la surface d'un matériau (en Bq. $\mathrm{m}^{-2} \cdot \mathrm{s}^{-1}$ ou, mieux, en atome. $\mathrm{m}^{-2} \cdot \mathrm{s}^{-1}$ ) dépend donc du coefficient de diffusion du radon 222 dans ce matériau (lui-même variable selon la porosité et l'humidité) et des forces convectives induites par des gradients de température, de pression,...

Ainsi, le flux d'émanation de radon 222 du sol, qui est en moyenne de $2.4 .10^{-2}$ Bq. $\mathrm{m}^{-2} \cdot \mathrm{s}^{-1}\left(10^{4}\right.$ atome. $\left.\mathrm{m}^{-2} \cdot \mathrm{s}^{-1}\right)$ diminue fortement en période de pluie, de neige, ou lorsque la pression atmosphérique augmente. De même, le flux d'émanation de radon 222 des murs d'une habitation est susceptible de varier de façon inversement proportionnelle à la pression atmosphérique $[5,8]$.

La mesure du flux d'émanation du radon 222 est un indicateur pratique et réaliste de la capacité d'une source à libérer du radon dans les circonstances du moment.

\subsubsection{L'interface source-milieu intérieur}

Un des aspects, peut-être le plus important, de l'évaluation de la contribution des sources est de déterminer de quelle manière ou par quelle voie elles participent effectivement à l'entrée de radon 222 dans rhabitation. 
Dans l'exemple le plus simple, qui peut être celui d'un appartement situé en étage, le radon 222 ne provient pratiquement que des parois du local. II suffit de mesurer le flux d'émanation de radon 222 en un nombre suffisant de points pour rendre compte de la nature composite des matériaux et de l'influence des agents de recouvrement.

Cependant, dans le cas d'habitations basses (ou aux étages inférieurs des bâtiments), les teneurs observées supposent très souvent une contribution non négligeable du sol. L'importance de cette contribution est fonction des caractéristiques architecturales de l'habitation, des possibilités de diffusion à travers les éléments du soubassement et des forces convectives créées à ce niveau par un gradient de température ou de pression.

En règle générale, la diffusion de radon 222 à travers les dalles séparant l'espace habitable des structures sous-jacentes est très largement favorisée par la présence de fissures ou d'ouvertures. Ce transfert peut s'accroître considérablement en présence d'un flux d'air ascendant induit par la différence de température entre l'intérieur et l'extérieur de la maison, différence qui favorise l'entrée d'air au niveau des parties basses de l'édifice (effet cheminée). Un gradient de pression sol-intérieur de la maison exerce le même effet.

Les données expérimentales dans ce domaine sont encore insuffisantes pour que l'on puisse préciser l'importance relative de ces transferts selon le type d'habitat, la nature des sols ou encore les conditions météorologiques. Quelques études récentes ont souligné la part dominante du sol dans le cas d'habitations individuelles [1, 4].

\section{RESULTATS D'UNE ETUDE PILOTE DE TRANSFERT DU RADON 222 DANS DEUX HABITATIONS DE LA VALLEE DU RHONE}

En aval du programme de surveillance [14], un protocole expérimental a été testé pour chercher à préciser les variables explicatives de certains cas d'augmentation de la concentration en radon 222 dans des habitations. L'étude porte sur deux maisons individuelles.

\subsection{Sélection de deux habitations}

Des dosimètres passifs ouverts (détecteurs LR 115), analogues à ceux utilisés dans les mesures systématiques, sont distribués périodiquement dans 131 habitations d'une aire géographique d'environ $3000 \mathrm{~km}^{2}$. La zone prospectée, caractérisée par la prédominance de sols sédimentaires, couvre une partie des départements de l'Ardèche, de la Drôme, du Gard et du Vaucluse. Chaque mesure est assortie d'un questionnaire détaillé relevant les caractéristiques de l'habitat, des pièces étudiées, des habitudes de vie des occupants,...

$\mathrm{Ce}$ lot de maisons individuelles présente des teneurs en radon 222 relativement faibles; les plus élevées observées pendant l'hiver sont comprises entre 100 et $230 \mathrm{~Bq} \cdot \mathrm{m}^{-3}\left(48.10^{6}\right.$ et $110.10^{6}$ atome. $\left.\mathrm{m}^{-3}\right)$ dans 10 maisons sur 107 explorées pendant cette période. Les relevés des autres saisons, moins nombreux, mettent en évidence des teneurs plus basses, soit 
4-70 Bq. $\mathrm{m}^{-3}\left(1,9.10^{6}-33.10^{6}\right.$ atome. $\left.\mathrm{m}^{-3}\right)$ au printemps (39 maisons) et 4-166 Bq. $\mathrm{m}^{-3}\left(1,9.10^{6}-79.10^{6}\right.$ atome. $\left.\mathrm{m}^{-3}\right)$ en été (53 maisons).

Dans une même habitation, les valeurs printanières semblent inférieures aux valeurs hivernales, particulièrement lorsque ces dernières dépassent 50 Bq. $\mathrm{m}^{-3}\left(24.10^{6}\right.$ atome. $\left.\mathrm{m}^{-3}\right)$. Par contre, les valeurs estivales sont tantôt inférieures, tantôt supérieures aux valeurs hivernales, probablement, selon que l'habitation est occupée ou non (fig. 1).
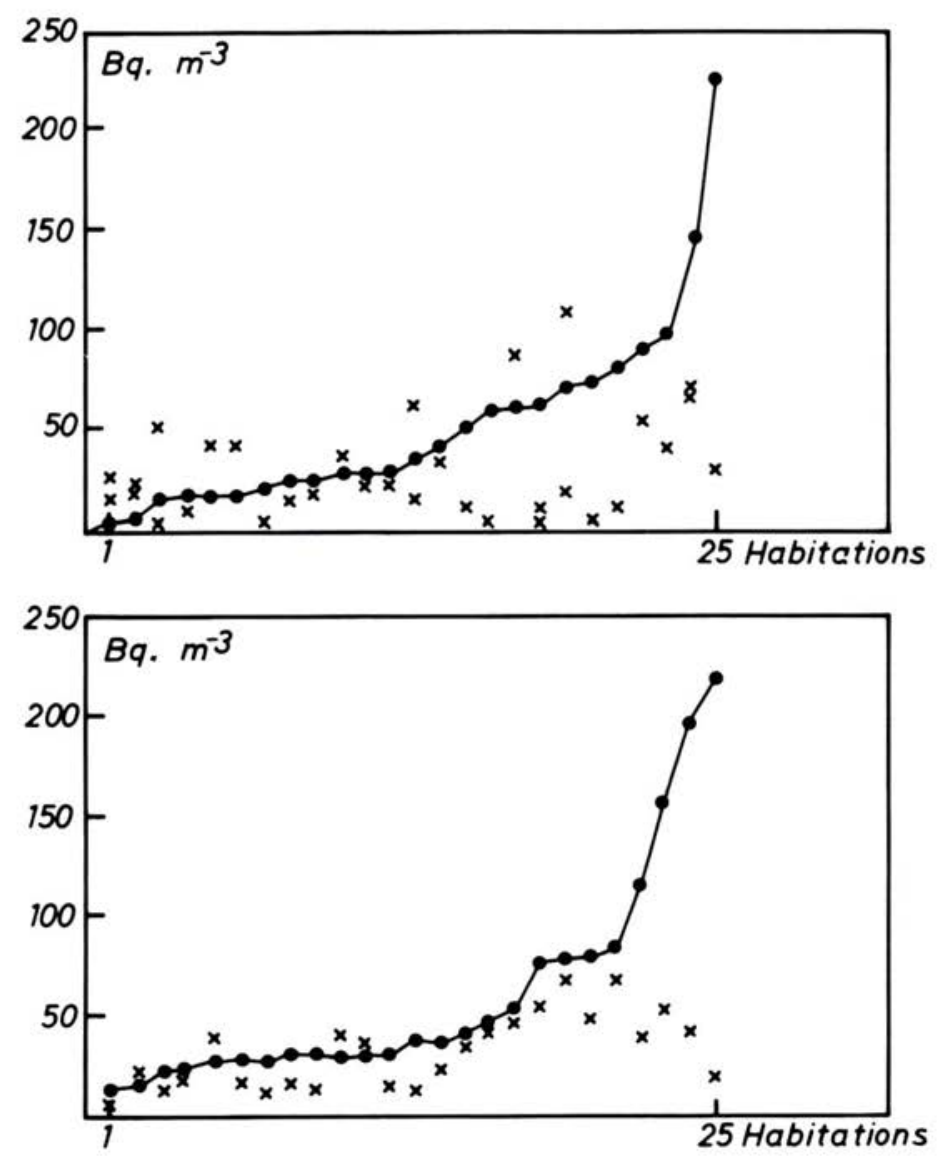

Fig. 1. - Comparaison des teneurs en radon. En haut: Hiver $(\bullet)-$ Eté (x). En bas: Hiver $(\bullet)$ - Printemps (x). 
Bien que portant sur un échantillon restreint, cette observation suggère que seules des mesures hivernales pourraient détecter de façon efficace des habitations à forte teneur en radon 222. Cette hypothèse explique l'attitude de certaines équipes suédoises qui réservent leur prospection à la saison de chauffage compte tenu des difficultés d'interprétation des relevés d'autres saisons [19].

Aucune variation significative au niveau du radon 222 n'est mise en évidence selon la nature du soubassement de la construction, l'étage ou l'usage des pièces.

Au terme de cette enquête, deux habitations traditionnelles en parpaings, élevées sur vide sanitaire et situées à moins de $100 \mathrm{~m}$ l'une de l'autre, sont sélectionnées parmi celles présentant les teneurs en radon 222 les plus élevées. Leur principales caractéristiques sont décrites dans le tableau II.

TABLEAU ॥

Principales caractéristiques des deux habitations examinées

\begin{tabular}{|c|c|c|c|}
\hline & Habitation A & \multicolumn{2}{|c|}{ Habitation B } \\
\hline $\begin{array}{l}\text { Architecture: } \\
\text { Année de construction } \\
\text { Matériaux: } \\
\text { - murs } \\
\text { - cloisons } \\
\text { Isolation des ouvertures } \\
\text { Plancher } \\
\text { Vide sanitaire }\end{array}$ & $\begin{array}{c}1980 \\
\text { parpaings, liège } \\
\text { briques, plâtre } \\
+++ \\
\text { hourdis, carrelage } \\
\text { ventilé }\end{array}$ & \multicolumn{2}{|c|}{$\begin{array}{c}\text { parpaings, brique, pierre } \\
\text { briques, plâtre } \\
+ \\
\text { hourdis, carrelage } \\
\text { non ventilé }\end{array}$} \\
\hline $\begin{array}{l}\text { Pièces étudiées: } \\
\text { Type } \\
\text { Niveau } \\
\text { Volume }\left(\mathrm{m}^{3}\right) \\
\text { Orientation des ouvertures } \\
\text { - Nord } \\
\text { - Sud } \\
\text { - Est } \\
\text { - Ouest }\end{array}$ & $\begin{array}{l}\text { salle de séjour } \\
\qquad \begin{array}{c}\text { Rdc } \\
270 \\
1 \\
3 \\
-\end{array}\end{array}$ & $\begin{array}{c}\text { S. de séjour } \\
1^{\text {er étage }} \\
110 \\
- \\
\frac{1}{2}\end{array}$ & $\begin{array}{l}\text { s. commune } \\
\text { Rdc } \\
90 \\
- \\
- \\
-\end{array}$ \\
\hline
\end{tabular}

\subsection{Etude de la variation de la concentration en radon 222 de l'air intérieur}

Le dispositif expérimental, déployé pendant trois jours consécutifs dans chacune des deux maisons, assure une mesure continue du radon 222 (chambre d'ionisation différentielle CD 43, 120 I, seuil de mesure : $10 \mathrm{~Bq} \cdot \mathrm{m}^{-3}$ ) et du débit d'air entrant dans les pièces examinées (méthode de traçage par le $\mathrm{SF}_{6}$, généré le long d'un capillaire disposé autour de la pièce) (fig. 2). L'air est prélevé en un point situé à 1,5-2 $\mathrm{m}$ du plancher, sous un débit suffisamment faible $\left(3 \mathrm{~m}^{3} \cdot \mathrm{h}^{-1}\right)$ pour ne pas modifier l'aération naturelle des pièces. 


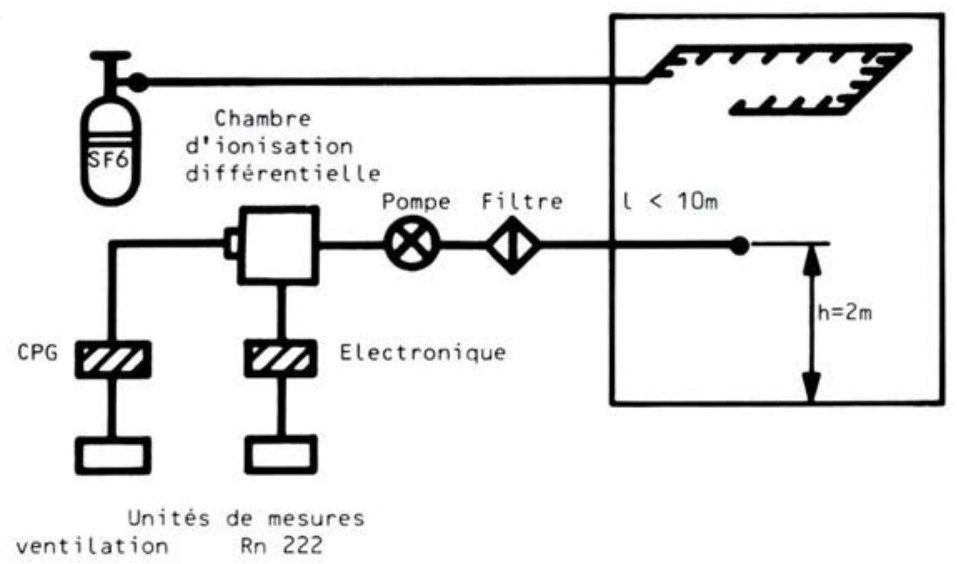

Fig. 2. - Dispositif expérimental

En parallèle, la concentration de radon 222 de l'atmosphère extérieure est enregistrée de façon continue à proximité immédiate des deux habitations. Les principales variables météoroloqiques sont relevées à partir d'un mât situé à moins de $3 \mathrm{~km}$ de distance (vitesse et direction du vent au niveau $12 \mathrm{~m}$, température et humidité au niveau $3,4 \mathrm{~m}$, pression au niveau $1,5 \mathrm{~m}$, gradient de température entre les niveaux $3,4 \mathrm{~m}$ et $101,4 \mathrm{~m}$ et, enfin, pluviométrie). Compte tenu de l'orographie du site et de la situation des deux maisons par rapport à ce mât, l'implantation locale d'un mât supplémentaire ne s'est pas avérée nécessaire. Enfin, les variations intérieures de température, de pression et d'humidité sont relevées dans les différentes pièces examinées.

Les deux habitations se distinguent l'une de l'autre par un important écart de ventilation. Le taux moyen de renouvellement de l'air est en effet en moyenne dix fois moindre dans l'habitation A dont les portes et les fenêtres sont équipées d'un système d'isolation. La teneur moyenne en radon 222 de l'air y est plus élevée que dans l'habitation $B$, dans laquelle elle est trois fois plus élevée au rez-de-chaussée qu'au $1^{\text {er }}$ étage. On note qu'au niveau de ce $1^{\text {er }}$ étage, la concentration moyenne de radon 222 est du même ordre que celle mesurée simultanément à l'extérieur (première journée) (tableau III).

\section{TABLEAU III}

Radon 222 et ventilation (moyennes)

\begin{tabular}{|c|c|c|c|c|c|c|}
\hline \multirow[b]{3}{*}{$\begin{array}{l}\text { Durée d'enregis- } \\
\text { trement (h) }\end{array}$} & \multirow{2}{*}{ Maison A } & \multicolumn{2}{|c|}{ Maison B } & \multicolumn{3}{|c|}{ Extérieur } \\
\hline & & Rdc & 1 er étage & $1^{e r} \mathrm{j}$ & $2^{e} j$ & $3^{e} \mathrm{j}$ \\
\hline & 72 & 40 & 30 & 15 & 10 & 12 \\
\hline $\begin{array}{l}\text { Concentration en } \\
\text { radon } 222 \text { de l'air } \\
\left(\mathrm{Bq} \cdot \mathrm{m}^{-3}\right)\end{array}$ & $\begin{array}{r}130 \\
+\quad 69\end{array}$ & $\begin{array}{r}89 \\
+\quad 47\end{array}$ & $\begin{array}{r}31 \\
+\quad 18\end{array}$ & $\begin{array}{r}24 \\
+\quad 13\end{array}$ & $\begin{array}{r}35 \\
+\quad 15\end{array}$ & $\begin{array}{r}39 \\
+\quad 14\end{array}$ \\
\hline $\begin{array}{l}\text { Taux de renouvel- } \\
\text { lement de l'air } \\
\left(\mathrm{h}^{-1}\right)\end{array}$ & $\begin{array}{r}0,28 \\
\pm 0,08\end{array}$ & $\begin{array}{r}1,85 \\
\pm 0,88\end{array}$ & $\begin{array}{r}1,95 \\
\pm 0,61\end{array}$ & & & \\
\hline
\end{tabular}


Les enregistrements (fig. 3) ne font apparaître de liaison nette entre les variables que dans le cas de l'habitation $A$ où la ventilation et la teneur en radon 222 de l'air semblent négativement corrélés; les deux courbes sont décalées de 2-3 heures l'une par rapport à l'autre. L'habitation A présente des variations significatives de température et d'humidité. A l'extérieur, la teneur en radon 222 de l'atmosphère augmente au cours des épisodes nocturnes d'inversion de température.

Les liaisons entre les niveaux de radon 222 et les variables intérieures ou extérieures aux habitations sont analysées de façon à rendre compte de la possibilité d'un délai dans l'effet de ces variables: les coefficients de corrélation entre les courbes sont calculés pour différents décalages horaires $(0,+1 \mathrm{~h},+2 \mathrm{~h},+3 \mathrm{~h})$. Les résultats mettent en évidence des paramètres explicatifs de la variation du niveau de radon 222 différents dans les deux habitations, tout au moins pour les trois jours de l'expérience (tableau IV).

TABLEAU IV

Valeur du coefficient de corrélation entre certaines variables

\begin{tabular}{|c|c|c|c|c|}
\hline \multicolumn{2}{|c|}{} & \multirow{2}{*}{ Maison A } & \multicolumn{2}{c|}{ Maison B } \\
\cline { 4 - 5 } & & Rdc & 1 er étage \\
\hline Taux de ventilation & & $r=-0,8$ & & \\
\hline $\begin{array}{c}\text { Température } \\
\text { et } \\
\text { humidité }\end{array}$ & intérieures & $r=-0,7$ & & \\
\cline { 4 - 5 } & extérieures & & $r=-0,9$ & $r=\begin{array}{r}0,9 \\
+0,9\end{array}$ \\
\hline Radon 222 extérieur & & & & $r=+0,8$ \\
\hline
\end{tabular}

\section{- Maison A}

La teneur en radon 222 de la pièce étudiée est négativement corrélée à la ventilation. L'effet de la variation du taux de renouvellement de l'air semble atteindre un maximum avec un retard de 3 heures. On observe également une liaison de la teneur en radon avec les variations de température et d'humidité intérieures, ces effets s'exerçant avec un décalage de l'ordre de 2 heures. Parmi les variables extérieures, seules la température et l'humidité (de toute évidence liées entre elles) semblent influer sur le niveau de radon à l'intérieur de la maison.

\section{- Maison B}

On observe de liaison entre la ventilation et la teneur en radon ni dans la pièce située au rez-de-chaussée ni dans celle du premier étage. Le taux de renouvellement de l'air de ces pièces est relativement élevé et la durée de l'observation est relativement courte dans les deux exemples. La température extérieure semble exercer une influence sur le niveau de radon 222 dans les deux étages. Par contre, seule la teneur en radon 222 du premier étage est fortement corrélée à la teneur en radon 222 de l'air extérieur. 

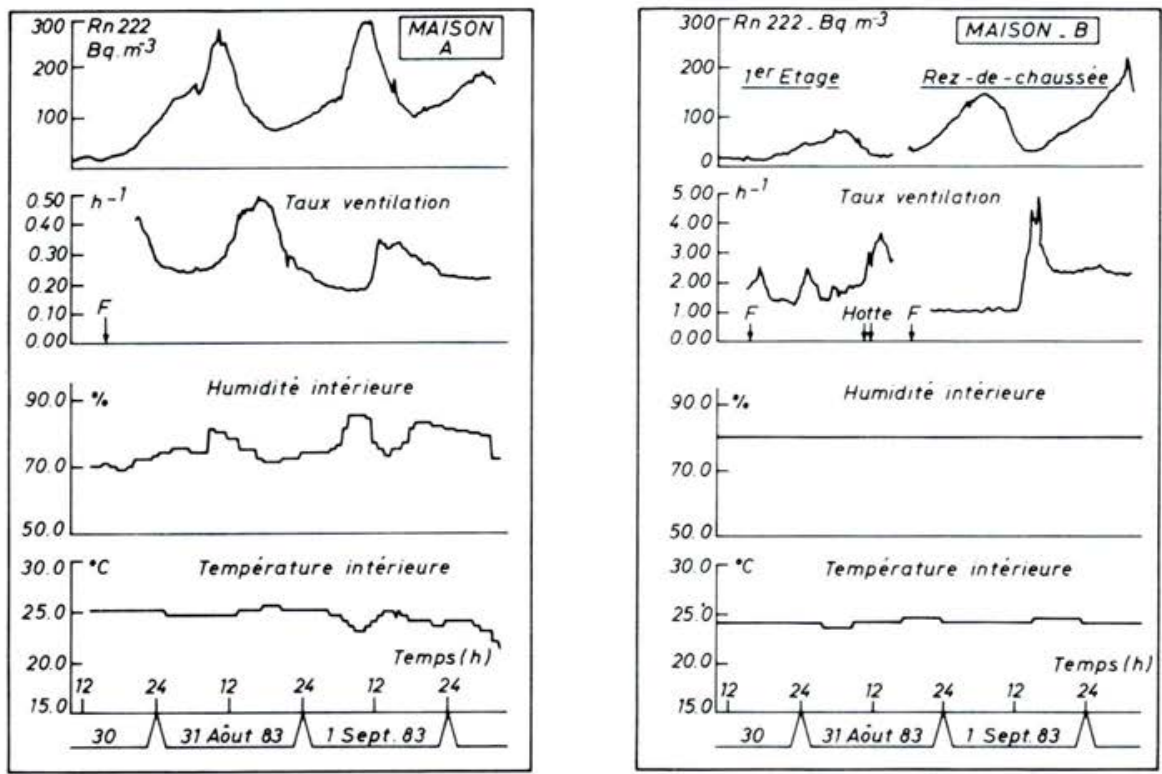

Variables intérieures
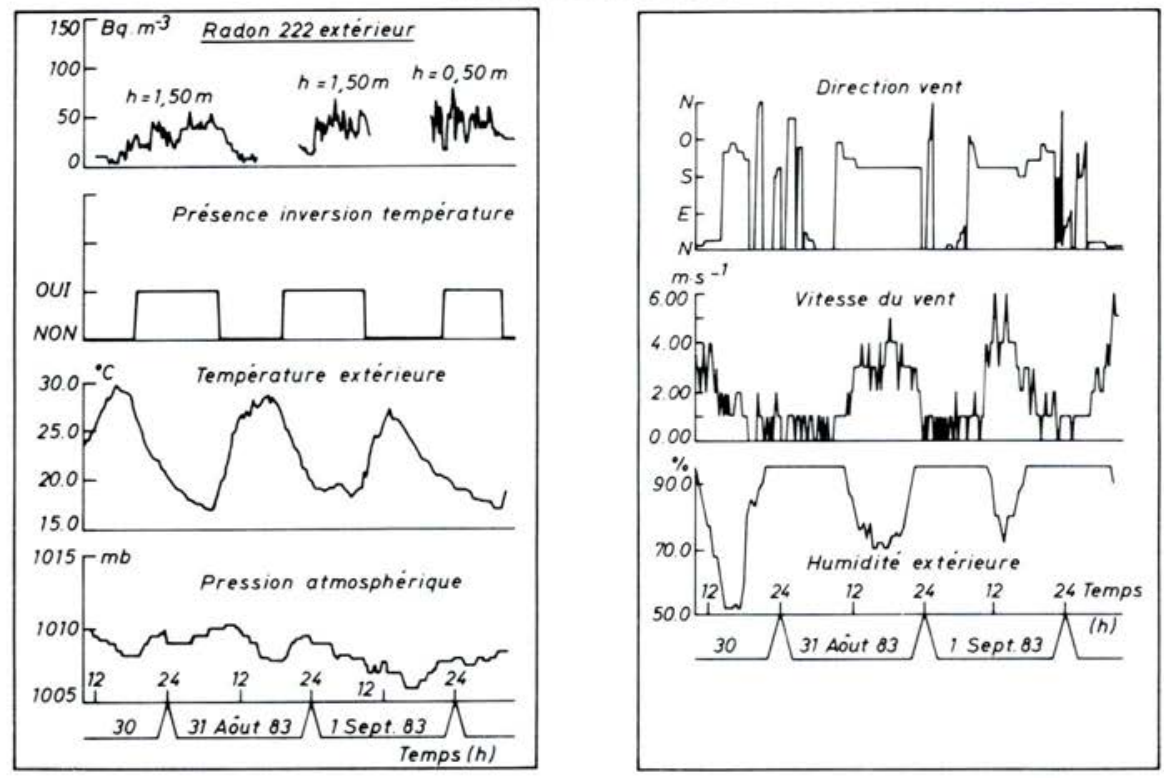

\section{Variables extèrieures}

Fig. 3. - Enregistrement des variables pendant 3 jours consécutifs. 
Aucune liaison entre la teneur en radon de l'air et la vitesse ou la direction du vent n'est mise en évidence dans le cas de la maison A qui ne possède pas d'ouverture à l'Ouest (direction dominante des vents dans la journée). Au contraire, la ventilation du premier étage de la maison B, possédant de grandes ouvertures à l'Ouest, dépend de la vitesse du vent $(r=0,8)$.

Au total, les paramètres intérieurs (température, humidité) induisent des variations de concentration de radon 222 en cas de faible ventilation naturelle (maison A). En cas de ventilation importante (maison B), les paramètres extérieurs constituent les principales variables explicatives au niveau du radon.

\subsection{Etude des sources}

Le flux d'émanation du radon 222 provenant des sols avoisinant les constructions ainsi que du sol du vide sanitaire de la maison A (le seul accessible) est déterminé en plusieurs emplacements par une méthode d'accumulation (bidon de 28 । de volume, diamètre $=0,3 \mathrm{~m}$ ). Par une méthode similaire, on mesure le flux d'émanation de radon 222 des dalles en béton des garages (au niveau et à côté des fissures apparentes), des parois non recouvertes d'enduits et, enfin, d'échantillons de matériaux de construction. Ces flux se situent dans la gamme des valeurs couramment observées $[7,20]$; ils apparaissent dix fois plus importants au niveau des sols qu'au niveau des surfaces intérieures où ils sont plus élevés en présence de fissures (fig. 4).

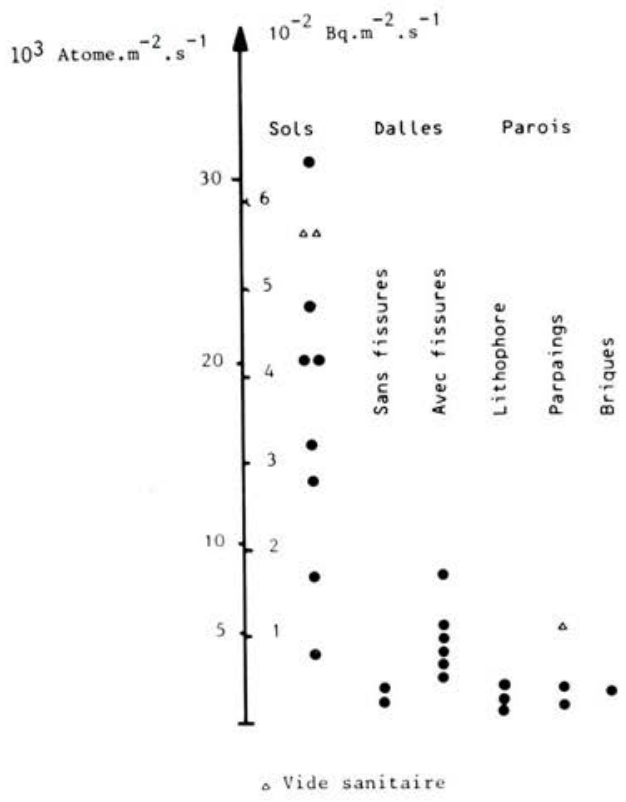

Fig. 4. - Flux d'émanation de radon 222. 
La concentration en radon des eaux de forage est identique à celle des eaux de distribution courante $\left(31.10^{3} \mathrm{~Bq} \cdot \mathrm{m}^{-3}\right.$, niveau relativement bas).

A ce point de l'étude, on peut tenter d'évaluer la contribution des sources en calculant la concentration de radon 222 dans l'air des pièces à partir du flux d'émanation des parois, de leur surface et du débit de renouvellement d'air des locaux. Les résultats de ces calculs concordent avec les mesures: selon les valeurs considérées pour chacun des paramètres, la concentration théorique est de 20 à 180 Bq. $\mathrm{m}^{-3}\left(9,6.10^{6}\right.$. $86,4 \cdot 10^{6}$ atome. $\mathrm{m}^{-3}$ ) pour la maison $\mathrm{A}$ (valeur mesurée $=130 \pm 69 \mathrm{~Bq} \cdot \mathrm{m}^{-3}$ ), 8 à 20 Bq. $\mathrm{m}^{-3}\left(3,8 \cdot 10^{6}-9,6 \cdot 10^{6}\right.$ atome. $\left.\mathrm{m}^{-3}\right)$ pour le premier étage de la maison $B$ (valeur mesurée $=31 \pm 18$ Bq. $\mathrm{m}^{-3}$ ) et 50 Bq. $\mathrm{m}^{-3}\left(24.10^{6}\right.$ atome. $\mathrm{m}^{-3}$ ) au maximum pour le rez-de-chaussée de la maison $B$ (valeur mesurée $=89 \pm 47$ Bq. $\mathrm{m}^{-3}$ ).

Dans les deux habitations, une part importante du radon 222 semble provenir du soubassement de la construction. Cette hypothèse est en partie confirmée par l'enregistrement complémentaire de la teneur en radon 222 du vide sanitaire de la maison $A$, qui atteint $2,3.10^{3} \mathrm{~Bq} \cdot \mathrm{m}^{-3}$ $\left(1,1.10^{9}\right.$ atome. $\left.\mathrm{m}^{-3}\right)$ lorsque toutes les bouches d'aération sont fermées. Communiquant avec l'espace habitable par l'intermédiaire d'une trappe, ce vide sanitaire est d'autant plus susceptible de contribuer à l'exposition domestique qu'il est moins ventilé. La durée de l'observation est toutefois trop brève pour vérifier cette hypothèse. Quelques auteurs [10, 15] ont montré l'importance des liaisons entre le vide sanitaire et l'espace habité et des mécanismes parfois originaux qui les régissent.

Ainsi, dans l'exemple d'une habitation de l'Oregon [11], les fluctuations du niveau de radon 222 à l'intérieur de l'habitation suivent celles du vide sanitaire; en période de pluie, davantage de radon pénètre dans la maison lorsque la pression atmosphérique chute, le soubassement de la construction devenant alors, selon les auteurs, la seule voie de sortie des gaz du sol.

\section{UNE RETOMBEE POSSIBLE DE L'ETUDE DU TRANSFERT DU RADON : L'ETUDE DE SYSTEMES DE LIMITATION}

Les sources étant identifiées et les mécanismes de transfert du radon 222 précisés, le dispositif expérimental décrit ci-dessus peut être utilisé pour contrôler l'efficacité de moyens de limitation. Une panoplie de contre mesures sont en effet décrites dans la littérature : recouvrement des parois par des papiers peints doublés de plastiques étanches, ou par des peintures isolantes, ventilation mécanique de l'espace habité, colmatage des fissures et des ouvertures des dalles, diminution de la pression au niveau du soubassement.

Deux exemples empruntés à des auteurs suédois [6] montrent la nécessité de tester l'efficacité de ces moyens :

\section{- 1er exemple (tableau V)}

Dans une maison individuelle où le radon 222 provient en partie des matériaux de construction et en partie du sol, la mise en œuvre d'un système de ventilation mécanique s'avère insuffisante pour réduire la concen- 
tration de radon 222 à un niveau jugé acceptable dans ce pays. La création d'un espace ventilé longitudinalement entre le plancher et la dalle se révèle très efficace.

TABLEAU V

Limitation de concentration de radon 222

\begin{tabular}{|c|c|c|c|c|}
\hline & & $\begin{array}{l}\text { Etat } \\
\text { initial }\end{array}$ & $\begin{array}{l}\text { Ventilation } \\
\text { forcée }\end{array}$ & $\begin{array}{l}\text { Ventilation } \\
\text { sous plancher }\end{array}$ \\
\hline $\begin{array}{l}\text { Salle de } \\
\text { jeux }\end{array}$ & $\begin{array}{c}R n^{*}\left(\text { Bq. } m^{-3}\right) \\
\text { Ventilation }\left(h^{-1}\right)\end{array}$ & $\begin{array}{c}1575-1600 \\
0,21\end{array}$ & $\begin{array}{c}1295 \cdot 1355 \\
0,21\end{array}$ & $\begin{array}{r}85-105 \\
0,49 \cdot 0,61\end{array}$ \\
\hline Séjour & $\begin{array}{c}R n^{*}\left(\mathrm{~Bq} \cdot \mathrm{m}^{-3}\right) \\
\text { Ventilation }\left(\mathrm{h}^{-1}\right)\end{array}$ & $\begin{array}{l}575 \cdot 1115 \\
0,20 \cdot 0,34\end{array}$ & $\begin{array}{l}455-1040 \\
0,31 \cdot 0,45\end{array}$ & $\begin{array}{c}165 \cdot 200 \\
0,51 \cdot 0,86\end{array}$ \\
\hline Cave & $\begin{array}{c}R n^{\star}\left(\text { Bq. } m^{-3}\right) \\
\text { Ventilation }\left(h^{-1}\right)\end{array}$ & ${ }_{0,6}^{680}-770$ & $\begin{array}{c}305-310 \\
0,93\end{array}$ & $\begin{array}{c}205 \cdot 235 \\
0,72\end{array}$ \\
\hline
\end{tabular}

- Concentration en descendants du radon 222.

- 2e exemple (fig. 5)

L'habitation est élevée sur un sol fortement émetteur en radon 222 (présence de schistes alunifères). La contribution des matériaux de construction est pratiquement négligeable. L'installation d'un système de drainage et de ventilation forcée, créant une dépression sous la dalle, permet de diminuer efficacement le niveau de radon 222 dans l'habitation (tableau VI).

TABLEAU VI

Limitation de concentration de radon 222

\begin{tabular}{|c|c|c|}
\hline \multicolumn{3}{|c|}{ Descendants du radon $222\left(\mathrm{~Bq} \cdot \mathrm{m}^{-3}\right)$} \\
\hline & Cave/salle de jeux & Séjour \\
\hline Etat initial & 1890 & 660 \\
\hline Après drainage & 230 & 185 \\
\hline
\end{tabular}

\section{CONCLUSION}

Cette courte étude pilote a permis de vérifier la validité d'un protocole. Elle montre la nécessité d'observations suffisamment prolongées pour mettre en évidence l'influence de certaines variables, voire, le cas échéant, pour tenter de tester l'efficacité de certains moyens de limitation (ventilation forcée du vide sanitaire, colmatage des fissures ou de la trappe d'accès au vide sanitaire de l'habitation A, quoique les niveaux de radon 222 ne justifient certainement pas de telles mesures). Le matériel utilisé dans cette expérience fonctionnant de façon quasi autonome, on pourra, dans un avenir proche, prolonger cette étude sur des habitations à forte teneur en radon 222 sélectionnées par les enquêtes systématiques. 


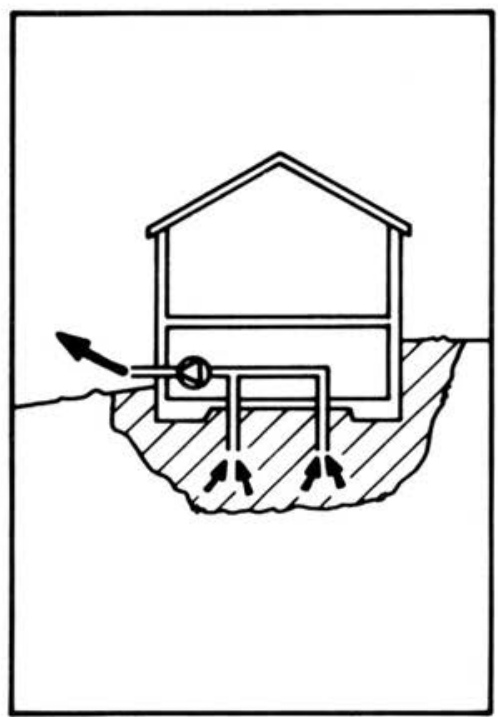

Fig. 5. - Système de limitation, d'après O. Hildingson, 1984.

\section{REFERENCES}

[1] AKERBLOM G., ANDERSON P., CLAVENSJO B. Soil gas radon - a source for indoor radon daughters. In : Indoor exposure to natural radiation and related risk assessment, Anacapri, 3-5 Oct. 1983. Radiat. Prot. Dos., 1984, 7 (1/4) 49-54.

[2] ALTER H.W., FLEISCHER R.L. Passive integrating radon monitor for environmental monitoring. Health Phys., 1981, 40, 693-702.

[3] DUPORT P., MADELAINE G., PINEAU J.F., ZETTWOOG P. Enregistrement des rayonnements alpha dans le dosimètre individuel et le dosimètre de site du Commissariat à l'énergie atomique. In : $10^{e}$ conférence internationale sur les détecteurs solides de traces nucléaires, Lyon, 2-6 juillet 1979 (FRANÇOIS H. et al., Eds). Oxford: Pergamon press, 1980, 609-615.

[4] EATON R.S., SCOTT A.G. Understanding radon transport in houses. In : Indoor exposure to natural radiation and related risk assessment, Anacapri, 3-5 Oct. 1983. Radiat. Prot. Dos., 1984, 7(1/4) 251-253.

[5] HERNANDEZ T.L., RING J.W., SACHS H.M. The variation of basement radon concentration with barometric pressure. Health Phys., 1984, 46. 440-445.

[6] HILDINGSON O., GUSTAFSSON J., NILSSON I. Locating and limiting radon in dwellings. In : Indoor exposure to natural radiation and related risk assessment, Anacapri, 3-5 Oct. 1983. Radiat. Prot. Dos., 1984, 7(1/4) 403.406.

[7] JONASSEN N. Exhalation measurements and indoor radon level in Denmark, Séminaire sur la charge radiologique de l'homme liée à la radioactivité naturelle dans les pays de la Communauté européenne. Le Vésinet, 4-6 décembre 1979. Luxembourg: CEC, 1980, 249-254.

[8] MC LAUGHLIN J.P., JONASSEN N. The effect of pressure drops on radon exhalation from walls. In : Natural radiation environment III, Houston, Apr. 1978. CONF - 780422. Springfield : NTIS, 1980, 1125-1226. 
[9] MILES J.C.H., DEW E.J. A passive radon gas detector for use in homes. In 11 th International conference on solid state nuclear track detectors, Bristol, Sept. 1981. Oxford: Pergamon press, 1982.

[10] NAZAROFF W.W. et al. Radon transport into a single-family house with a basement. LBL - 19572, 1984.

[11] NAZAROFF W.W., DOYLE S.M. Radon entry into houses having a crawl space. LBL - 16637, 1983.

[12] NERO A.V., NAZAROFF W.W. Characterising the source of radon indoors. In : Indoor exposure to natural radiation and related risk assessment, Anacapri. 3-5 Oct. 1983. Radiat. Prot. Dos., 1984, 7(1/4), 23-29.

[13] OCDE. Agence pour l'énergie nucléaire. Exposition aux rayonnements due à la radioactivité naturelle des matériaux de construction. Paris: OCDE, 1979.

[14] RANNOU A. et al. Résultats de surveillance du radon à l'intérieur des habitations. Les données actuelles sur la radioactivité naturelle. Congrès SFRP, Monte-Carlo, 5-7 novembre 1984.

[15] RUNDO J., MARKUN F., PLONDKE N.J. Observation of high concentration of radon in certain houses. Health Phys., 1979, 36, 729-730.

[16] SCIOCCHETTI G. and al. Indoor measurements of air-borne natural radioactivity in Italy. In : Indoor exposure to natural radiation and related risk assessment, Anacapri, 3-5 Oct. 1983. Radiat. Prot. Dos., 1984, 7(1/4), 347-351.

[17] STRANDEN E., BERTEIG L. Radon in dwellings and influencing factors. Health Phys., 1980, 39, 275-284.

[18] SWEDJEMARK G.A. Indoor measurements of natural radioactivity in Sweden. Séminaire sur la charge radiologique de l'homme liée à la radioactivité naturelle dans les pays de la Communauté européenne. Le Vésinet, 4-6 décembre 1979 Luxembourg : CEC, 1980, 271-295.

[19] SWEDJEMARK G.A. Temporal variations of the radon concentration indoors. In : Indoor exposure to natural radiation and related risk assessment, Anacapri, 3-5 Oct. 1983. Radiat. Prot. Dos., 1984, 7(1/4) 225-258.

[20] UNITED NATIONS SCIENTIFIC COMMITTEE on the effects of atomic radiation (UNSCEAR). Ionizing radiation: sources and biological effects. New York : Nations Unies, 1982, Annex D.

[21] PIESCH E., URBAN M. Low level environmental radon dosimetry with a track etch detector device. Radiat. Prot. Dos., 1981, 1, 97-109.

[22] ZETTWOOG P. La physique des processus de l'irradiation des travailleurs dans les exploitations minières. Club Vassivière (à paraître). 